\title{
Exploratory analysis of CD63 and CD203c expression in basophils from hazelnut sensitized and allergic individuals
}

\author{
Bianca Lötzsch ${ }^{1 \dagger}$, Sabine Dölle ${ }^{1 \dagger}$, Stefan Vieths ${ }^{2}$ and Margitta Worm ${ }^{1 *}$
}

\begin{abstract}
Background: Sensitization to hazelnut $(\mathrm{HN})$ is frequent and requires clarification to determine whether this sensitization is clinically relevant. The aim of this study was to investigate basophil activation profiles in $\mathrm{HN}$-sensitized and allergic subjects.
\end{abstract}

Methods: Basophil activation was determined by flow cytometric analyses of CD63 and CD203c expression using several HN allergen concentrations. Depending on their clinical reaction pattern, an oral allergy symptom group (OAS, $n=20)$, a systemic reaction group $(n=12)$ and a sensitized group without clinical symptoms $(n=20)$ were identified. Additionally, 10 non-allergic and non-sensitized individuals served as controls.

Results: CD63 and CD203c expression differed between allergic (OAS and systemic group) and sensitized subjects.

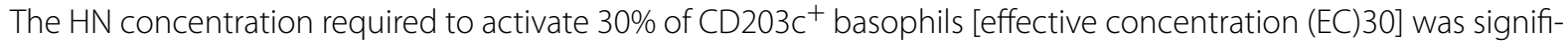
cantly higher in sensitized versus the allergic group $(p=0.0089)$. This was more pronounced when the basophil allergen threshold sensitivity (CD-sens) was calculated (CD63: $p=0.018 ; C D 203 c: p=0.009)$.

Conclusion: Our data indicate that the basophil activation test may provide information to better distinguish between sensitized and allergic subjects if several allergen concentrations are considered. CD203c expression displayed a better discrimination compared to CD63; therefore, its diagnostic value might be superior compared with CD63.

Keywords: Food allergy, Hazelnut, Basophil activation test, CD63, CD203

\section{Background}

In Europe, hazelnut (Corylus avellana) is a frequent cause of food allergy [1] with a prevalence of $0.1-0.5 \%$ [2]. In adults, hazelnut (HN) allergy is often associated with a pre-existing birch pollen allergy that can develop after inhalant sensitization with the major birch pollen allergen Bet $\mathrm{v} 1$, a pathogenesis-related protein of family 10 [3]. Homologous immunoglobulin (Ig)E-binding epitopes of the pollen-related major $\mathrm{HN}$ allergen Cor a 1 are responsible for cross reactivity to Bet v $1[4,5]$. In Central Europe, sensitization to Bet $\mathrm{v} 1$ is the major cause

*Correspondence: margitta.worm@charite.de

${ }^{\dagger}$ Bianca Lötzsch and Sabine Dölle contributed equally to this work.

${ }^{1}$ Department of Dermatology and Allergology, Allergy-Center-Charité, Charité - Universitätsmedizin Berlin, Charitéplatz 1, 10117 Berlin, Germany Full list of author information is available at the end of the article of pollen-associated food allergies [1]. Up to $50-90 \%$ of birch-pollen-allergic patients develop sensitivity to food such as HN, apple, and others [3].

In addition to Cor a 1, other HN allergens, namely, Cor a 2 (profilin), Cor a 8 (lipid transfer protein), Cor a 9 (legumin-like protein), Cor a 11 (vicilin-like protein) and Cor a 14 (2S albumin), have been described to cause $\mathrm{HN}$ sensitization [6-8].

Although, $\mathrm{HN}$ allergy frequently leads to oral allergic symptoms (OAS), it can also cause severe and even lifethreatening reactions $[9,10]$.

The diagnosis of food allergy mainly includes a detailed case history, skin prick testing (SPT) and the measurement of food-specific IgE (sIgE) [11, 12]. However, the low specificity of SPT and sIgE may cause over-diagnosis, which leads to unnecessary diet restrictions resulting in 
a lower quality of life [13]. Thus, the diagnosis of a food allergy should be proven by double-blind, placebo-controlled oral food challenges (DBPCFC) [11, 12]. Although, DBPCFC is the gold standard in diagnosing food allergies, it is not often included in daily evaluation for several reasons such as limited time and resources [14, 15].

Diagnostic tests that may support the discrimination between sensitized and symptomatic subjects without the high risk of developing an anaphylactic reaction are desirable. The basophil activation test (BAT) is an in vitro test that determines the expression of defined basophil markers (CD63 and CD203c) after allergen activation. The BAT has been suggested as a useful tool for the diagnosis of different IgE-mediated allergies [16].

The aim of this study was to investigate the CD63 and CD203c activation profiles in sensitized and symptomatic $\mathrm{HN}$-allergic subjects considering several $\mathrm{HN}$ allergen concentrations. Moreover, we correlated the data from these surface markers with diagnostic parameters (SPT and SIgE), which are the most frequently used.

\section{Methods \\ Subjects}

Subjects were recruited from the Allergy-Centre-Charite (Berlin, Germany). Four groups (control, sensitized, OAS, systemic) were stratified based on case history including a detailed questionnaire about their symptoms after ingesting HN and the SPT data. Symptoms like itching or swelling of the oral mucosa (lips, tongue, palate), as well as throat tightness and dysphagia were counted for OAS. The following symptoms were considered as systemic: dyspnea, vomiting, emesis, diarrhea, generalised urticaria, general erythema, angioedema, as well as cardio-vascular symptoms, and rhinitis/rhinoconjunctivitis. Exclusion criteria were pregnancy, lactation, the use of antihistamines, and immunomodulating or immunosuppressive drugs. The study was approved by the local ethics committee of Charité (EA-No.: 1832/Si.258). All subjects gave written informed consent to participate in the study.

\section{Skin prick test (SPT)}

A SPT was performed (according to the recommendations [17]) with birch extract (Alk-Abelló, Wedel, Germany) and with fresh food (HN, apple, celery, carrot) by using the prick-to-prick method. Histamine dihydrochloride $(10 \mathrm{mg} / \mathrm{ml}$, ALK-Abelló) and sodium chloride $(0.9 \%$ $\mathrm{NaCl}$, ALK-Abelló) served as positive and negative controls. SPT was considered positive if the wheal diameter was $\geq 3 \mathrm{~mm}$ after $15 \mathrm{~min}$.

\section{Total and specific immunoglobulin E}

The serum samples were stored at $-20{ }^{\circ} \mathrm{C}$ until use. The measurement of the total and $\operatorname{SIgE}$ to $\mathrm{rBet} \mathrm{v} 1$, rCor a
1.04 (hereinafter referred to as rCor a 1) and rCor a 8 were determined with the ImmunoCAP System (Thermo Fisher Scientific, Uppsala, Sweden) according to the manufacturer's instructions. In addition, Cor a 9, Cor a 11 and Cor a 14 were measured by the Paul-Ehrlich-Institute (Langen, Germany).

\section{Basophil activation test (BAT)}

For measurement of basophil activation, heparinized blood samples were taken from subjects and analyzed within $24 \mathrm{~h}$. The blood samples were stimulated with increasing concentrations $\left(10^{-6}-10 \mu \mathrm{g} / \mathrm{ml}\right)$ of $\mathrm{HN}$ extract (European hazelnut extract, Greer Labs, Lenoir, NC, USA) and incubated for $15 \mathrm{~min}$ at $37^{\circ} \mathrm{C}$. Anti-human IgE (BIOZOL HP6029/HP6061, Eching, Germany) was used as positive, and RPMI 1640 medium (Bio Chrom AG, Berlin, Germany) was used as a negative control. Cells were stained with anti-CD63-FITC (BD Biosciences, Franklin Lakes, NJ, USA), anti-CD203c-PE (Immunotech Inc., Canada), anti-CD3-VioBlue (Myltenyi Biotec GmbH, Bergisch-Gladbach, Germany) and anti-CCR3APC (R\&D Systems, Minneapolis, MN, USA). After adding lysing solution (BD Biosciences, Franklin Lakes, NJ, USA), the basophils were analyzed by flow cytometry (Miltenyi MACS Quant Flow Cytometer, BergischGladbach, Germany). $\mathrm{CD}^{-} / \mathrm{CCR} 3^{+}$cells were gated as basophils, $\mathrm{CD}^{-} / \mathrm{CCR}^{+} / \mathrm{CD} 203^{\text {high }}+$ cells were analyzed for basophil activation, and $\mathrm{CD}^{-} / \mathrm{CCR} 3^{+} / \mathrm{CD} 3^{+}$cells were defined as degranulated basophils. The data were analyzed using the MACSQuantify Software ${ }^{\mathrm{TM}}$ program (Miltenyi Biotec $\mathrm{GmbH}$ ). Expression values were normalized for basophil activation induced by anti-IgE. The analysis of CD203c expression was based on the allergen concentration, which activated $30 \%$ of $\mathrm{CD}_{203 \mathrm{c}^{+}}$basophils [effective concentration (EC)30] as previously performed [18]. For CD63, the maximal percentage of CD63 up-regulation at one allergen dose (CD-max) was measured. Additionally, the basophil allergen threshold sensitivity (CD-sens) and EC50 (the effective concentration giving $50 \%$ of maximum up-regulation) were calculated as described by Glaumann et al. [19]. Subjects with less than $15 \%$ change in $\mathrm{CD}^{-} / \mathrm{CCR}^{+} / \mathrm{CD}^{203} 3^{\text {high }+}$ or $\mathrm{CD}^{-} /$ $\mathrm{CCR}^{+} / \mathrm{CD} 3^{+}$expression between negative and positive control were regarded as non-responders and excluded from further analysis $(\mathrm{n}=5)$.

\section{Statistical analysis}

Statistical analysis was performed with IBM SPSS Statistics (Chicago, IL, USA) and Prism (GraphPad Software, San Diego, CA, USA). All results are shown as medians with ranges (min-max, unless otherwise stated). Differences between the groups were verified with the KruskalWallis test or Mann-Whitney U test for non-Gaussian 


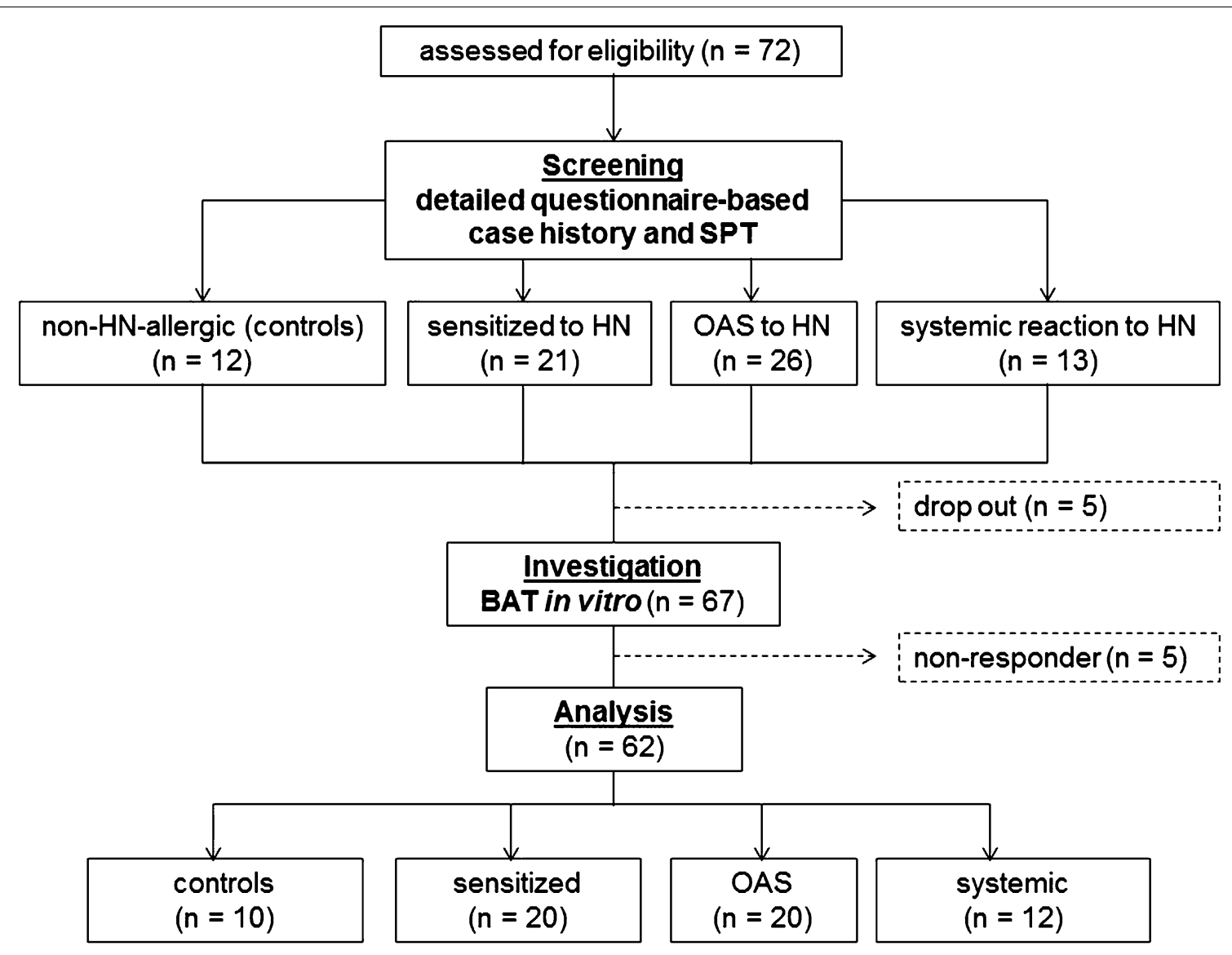

Fig. 1 Study flow chart and classification of subjects. HN hazelnut, SPT skin prick test, OAS oral allergic symptoms, BAT basophil activation test

distribution. The Spearman correlation coefficient was used for comparison between the diagnostic tests. $\mathrm{p}$ values $<0.05$ were considered to be statistically significant. Significant results should be regarded as descriptive and explorative due to the small sample size in this study.

\section{Results}

\section{Identification of subjects}

In total, 72 subjects were screened, and the BAT was performed in 67 individuals; of these, 62 were analyzed [median age: 31 (19-62) years, $\mathrm{n}=40$ (65\%) females and $\mathrm{n}=22$ (35\%) males; Fig. 1; Table 1]. Table 1 summarizes the subjects' characteristics. In this study, 10 subjects had a negative SPT for HN and birch and no symptoms when consuming HN (controls) and 20 subjects showed no symptoms but had a positive SPT for HN (sensitized). In addition, 32 subjects had a positive SPT for HN and according to their clinical symptoms, these were divided into subjects with OAS $(\mathrm{n}=20)$ and subjects with systemic reactions $(n=12)$.

\section{Sensitization profile}

SPT and sIgE were used to assess the sensitization status. All subjects from the sensitized, OAS and systemic groups had a positive SPT to HN (inclusion criteria). The wheal diameters for HN were not significantly different between the sensitized and symptomatic groups (Table 1). A similar pattern was observed when the birch pollen extract was used. In the symptomatic group, all 32 subjects, and in the sensitized group, 15 of 20 subjects, had a positive SPT to birch pollen extract (Table 1).

Total IgE levels were comparable among the sensitized, OAS and systemic groups and as expected, significantly higher compared to the control group (Table 1). In the sensitized group, the sIgE level against rCor a 1 [1.9 $(0.0-7.4) \mathrm{kU} / \mathrm{l}]$ was significantly lower compared to the sIgE level from the OAS group [6.7 (0.0-63.8) $\mathrm{kU} / \mathrm{l}]$, but not compared to the systemic group [3.5 (0.1-28.1 kU/l)]. For both symptomatic groups, higher ratios of sIgE to total IgE levels were calculated for Cor a 1 in comparison to the sensitized group. The ratios for $\mathrm{rBet} v 1$ were 
comparable between the sensitized and symptomatic groups (Table 1). No sensitization to Cor a 9, Cor a 11 or Cor a 14 were found.

\section{Basophil activation profiles}

The BAT was performed in 67 screened subjects; among these, 5 (3.35\%) were non-responders (Fig. 1).

The CD63 expression measured as basophil reactivity (maximal reactivity, CD-max) showed differences between clinically symptomatic (OAS/systemic) and sensitized subjects (Fig. 2a). The median CD63 expression in the sensitized group was lower for all $\mathrm{HN}$ extract concentrations than the median of CD63 expression of the subjects with symptomatic HN allergy. However, no significant differences were detected by comparing CDmax, which was measured at a concentration of $0.1 \mu \mathrm{g} /$ $\mathrm{ml}$ of $\mathrm{HN}$ extract in the groups (sensitized, OAS, systemic) (Fig. 2c). Additionally, EC50 and CD-sens for CD63 were calculated (Table 2). We detected a significant higher CD-sens in the symptomatic group: 44.73 (13.01-96.72; $\mathrm{p}=0.018$ ) compared to the sensitized group: 6.59 (0.07-57.84). However, we could not differentiate between the OAS and systemic groups.

For CD203c, the effective concentration by which $30 \%$ of basophils were activated (EC30) was calculated. Higher $\mathrm{HN}$ concentrations were required to achieve an increase of CD203c expression in sensitized subjects (Fig. 2b), which was significant when calculated for EC30 $(\mathrm{p}=0.0089$, Fig. $2 \mathrm{~d})$. In order to better compare, we calculated EC50 and CD-sens for CD203c too (Table 2). The measured differences between CD-sens of the symptomatic group: 80.33 (14.64-267.87) and the sensitized group: $10.03(3.55-82.90)$ were significant $(\mathrm{p}=0.009)$. Again, we could not differentiate between the OAS and systemic group.

\section{Correlations between SPT, slgE and BAT}

We identified statistical correlations $(\mathrm{p}<0.001)$ between the CD63 and CD203c expression values at single HN concentration versus SPT, sIgE to $\mathrm{rCor}$ a 1 , and SIgE to rBet $\mathrm{v} 1$. The highest correlation was detected for $0.01 \mu \mathrm{g} /$ $\mathrm{ml}$ and is summarized in Table 3. A correlation between

Table 1 Subjects' characteristics

\begin{tabular}{|c|c|c|c|c|c|}
\hline & Controls $(n=10)$ & Sensitized $(n=20)$ & OAS $(n=20)$ & Systemic $(n=12)$ & p values \\
\hline Age (median [min-max] in years) & $30[20-57]$ & 26 [19-60] & $32[21-51]$ & $40[24-62]$ & NS \\
\hline Male sex (\%) & $2(20 \%)$ & $12(60 \%)$ & $6(30 \%)$ & $2(17 \%)$ & $0.041^{c}$ \\
\hline \multicolumn{6}{|l|}{ Atopic history [number (\%)] } \\
\hline Allergic rhinitis & $3^{\mathrm{a}}(30 \%)$ & $14(70 \%)$ & $16(75 \%)$ & $9(75 \%)$ & \\
\hline Atopic dermatitis & $2(20 \%)$ & $5(25 \%)$ & $9(50 \%)$ & $5(33 \%)$ & \\
\hline Allergic asthma & - & $9(35 \%)$ & $10(55 \%)$ & $8(58 \%)$ & \\
\hline \multicolumn{6}{|l|}{ SPT (median [min-max] in mm) } \\
\hline Birch & $0^{*}$ & $6[0-12]$ & $7[0-11]$ & $9[3-11]$ & NS \\
\hline Hazelnut & $0^{*}$ & $4[3-8]$ & $5[3-10]$ & $6[4-14]$ & NS \\
\hline Celery & $0^{*}$ & $0[0-7]$ & $3[0-10]$ & $4[0-7]$ & NS \\
\hline Apple & $0^{*}$ & $3[0-8]$ & $4[0-7]$ & $5[0-20]$ & NS \\
\hline Carrot & $0^{*}$ & $0[0-10]$ & $3[0-15]$ & $4[0-14]$ & NS \\
\hline \multicolumn{6}{|l|}{ IgE (median [min-max] in kU/l) } \\
\hline Total-IgE & $16[3-34]^{*}$ & 184 [20-2097] & $178[24-6861]$ & $59[26-4801]$ & NS \\
\hline slgE Bet $\vee 1$ & $0.0[0.0-0.1]^{*}$ & $3.5[0.0-16.2]$ & $10.2[0.0-72.4]$ & $5.9[0.1-43.6]$ & NS \\
\hline slgE Cor a 1 & $0.0[0.0-0.1]^{*}$ & $1.9[0.0-7.4]$ & $6.7[0.0-63.8]$ & $3.5[0.1-28.1]$ & $0.0254^{d}$ \\
\hline slgE Cor a 8 & n.d. & n.d. & $0.2[0.0-1.3]$ & $0.0[0.0-0.0]$ & NS \\
\hline \multicolumn{6}{|l|}{ Ratio $^{\mathrm{b}}$ (median [min-max]) } \\
\hline rBet v 1/total-lgE & $0.00^{*}$ & $0.04[0.00-4.19]$ & $0.07[0.00-13.0]$ & $0.04[0.00-11.9]$ & NS \\
\hline rCor a $1 /$ total-IgE & $0.00^{*}$ & $0.02[0.00-0.80]$ & $0.20[0.00-4.36]$ & $0.18[0.00-4.77]$ & $0.0226^{\mathrm{e}}$ \\
\hline
\end{tabular}

Statistical significances were analyzed with Kruskal-Wallis test

* Significant difference of the control group versus the other groups

a According to case history, not caused by birch pollen

b Calculated as slgE * slgE/total lgE

c Sensitized versus control and systemic group

d Sensitized versus OAS group

e Sensitized versus OAS and systemic group 
a

\section{CD63 dose-response curve (anti-lgE = 100\% CD63 expression)}

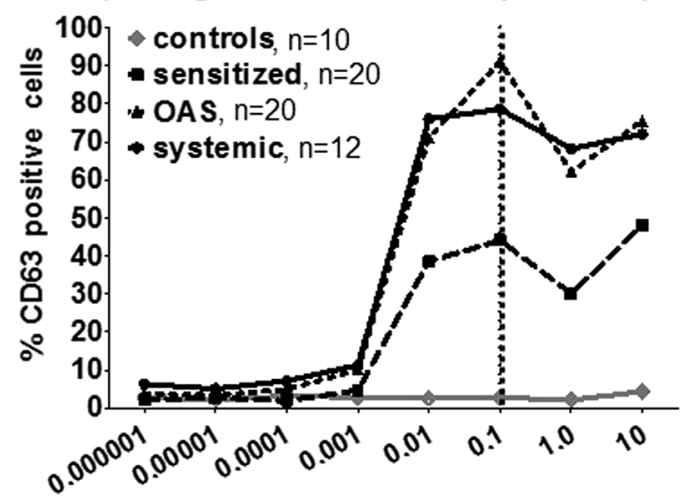

$\mathrm{HN}$ extract concentration in $\mu \mathrm{g} / \mathrm{ml}$

b

CD203c dose-response curve (anti-lgE $=100 \%$ CD203c expression)

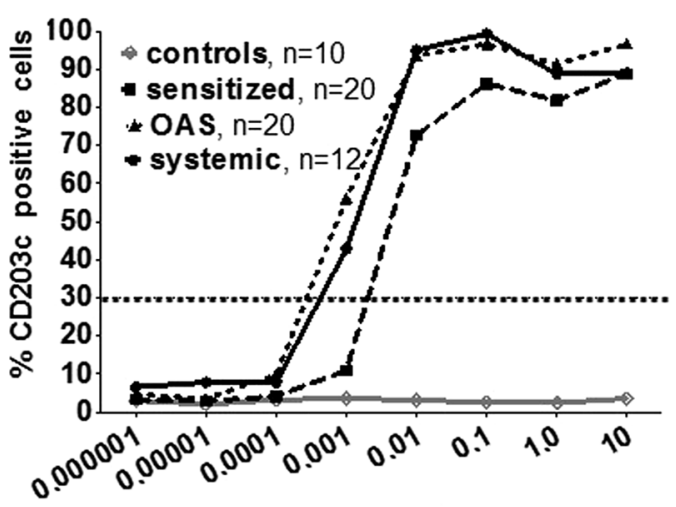

$\mathrm{HN}$ extract concentration in $\mu \mathrm{g} / \mathrm{ml}$
C

\section{CD-max for CD63 expression \\ at $0.1 \mu \mathrm{g} / \mathrm{ml} \mathrm{HN}$ extract \\ (anti-lgE $=100 \%$ )}

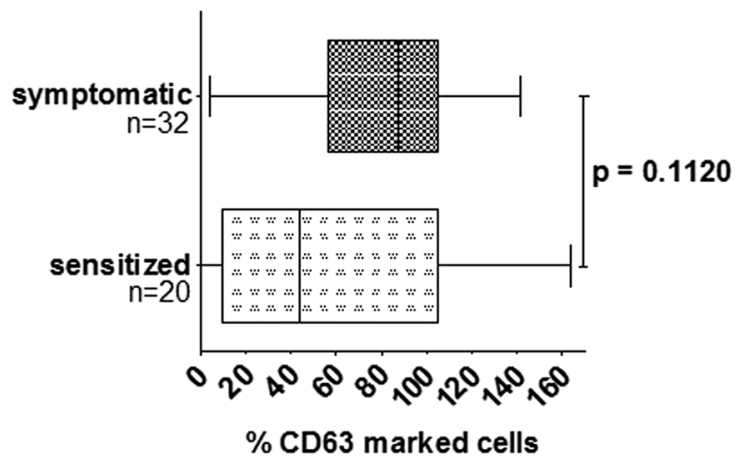

d

\section{EC30 for CD203c expression (anti-lgE $=100 \%$ )}

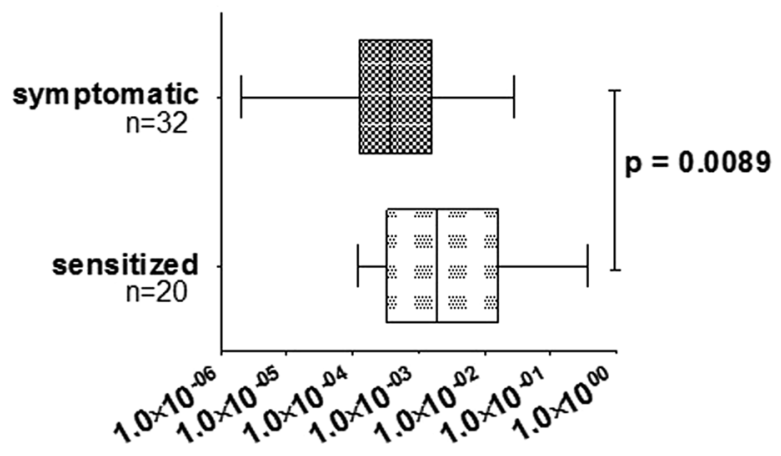

$\mathrm{HN}$ extract concentration in $\boldsymbol{\mu g} / \mathbf{m l}$

Fig. 2 Expression of CD63 and CD203c normalized for basophil activation induced by the positive control (anti-lgE). $\mathbf{a}$, b The dose-response curves for CD63 (a) and CD203C (b). The values are shown as medians. For a clear picture, the ranges are not shown in $\mathbf{a}$ and $\mathbf{b}$. The net values are depicted in Additional file 1: Figure S1 as medians with interquartile ranges for the four groups. The vertical dotted line indicates the maximum CD63 expression (CD-max) at the hazelnut (HN) concentration of $0.1 \mu \mathrm{g} / \mathrm{ml}(\mathbf{a})$, and the horizontal dotted line gives the concentration required to activate $30 \%$ of CD203c $\mathrm{C}^{+}$basophils [effective concentration (EC)30] (b). c, d The comparison between the sensitized $(n=20)$ and symptomatic (OAS and systemic, $\mathrm{n}=32$ ) groups by c Maximum CD63 expression (CD-max) at $0.1 \mu \mathrm{g} / \mathrm{ml}$ of hazelnut (HN) extract and $\mathbf{d}$ HN extract concentrations that activated $30 \%$ of basophils (EC30). Statistical significances were analyzed with Mann-Whitney $U$ test

CD-sens versus the other diagnostic measurements was only detected for CD203c (Table 3).

\section{Discussion}

Our results show that the BAT is a useful tool to determine biological activity against a food allergen such as HN. The correlation between CD203c/CD63 expression and the sensitization results measured by SIgE and SPT indicates basophil activity can be linked to sensitization status. Moreover, the CD203c-based BAT may distinguish between sensitized and symptomatic individuals but only if a careful dose-response analysis is performed.
The literature is controversial about the advantages and disadvantages of basophil surface markers [20]. CD63, compared to CD203c, is not a basophil-specific marker and has been shown to be less specific and less sensitive (in grass pollen and house dust mite allergy, latex allergy, wasp venom hypersensitivity, peanut allergy) [21-23]. On the other hand, the up-regulation of CD63 expression has been reported to provide similar results to CD203c expression in cat-allergic subjects [24] and to be more sensitive and specific in egg-allergic children [23]. All these data suggest a high variability of sensitivity and specificity depending on the allergen and cohort that was studied. 
Table 2 Basophil allergen threshold sensitivity (CD-sens) and half-maximal effective concentration (EC50)

\begin{tabular}{|c|c|c|c|c|c|c|c|}
\hline \multirow[t]{2}{*}{ Subject group } & \multirow[t]{2}{*}{ No. of subjects } & \multicolumn{3}{|l|}{ CD63 } & \multicolumn{3}{|l|}{ CD203c } \\
\hline & & EC50 (ng/ml) & CD-sens & $p$ values & $\mathrm{EC50}(\mathrm{ng} / \mathrm{ml})$ & CD-sens & $p$ values \\
\hline Sensitized & 20 & $15.18(1.73-1336)$ & $6.59(0.07-57.84)$ & & $10.09(1.21-33.38)$ & $10.03(3.55-82.90)$ & \\
\hline OAS & 20 & $2.24(1.05-7.12)$ & $44.73(14.07-96.38)$ & 0.028 & $0.97(0.17-6.95)$ & 104.55 (14.92-602.48) & 0.006 \\
\hline Systemic & 12 & $2.61(0.98-9.94)$ & $40.40(10.23-104.61)$ & NS & $1.74(0.55-7.95)$ & $59.35(13.27-190.12)$ & NS \\
\hline $\begin{array}{l}\text { Symptomatic (OAS + sys- } \\
\text { temic) }\end{array}$ & 32 & $2.24(1.04-7.70)$ & $44.73(13.01-96.72)$ & 0.018 & $1.27(0.37-7.02)$ & $80.33(14.64-267.87)$ & 0.009 \\
\hline
\end{tabular}

Values are given as median with interquartile range (IQR). Statistical significances were analyzed with Mann-Whitney $U$ test compared to the sensitized group

Table 3 Correlation of basophil activation test (BAT) with different diagnostic tests

\begin{tabular}{|c|c|c|c|c|}
\hline & \multicolumn{2}{|c|}{$\begin{array}{l}\text { Expression values } \\
\text { at } 0.01 \mu \mathrm{g} / \mathrm{ml} \mathrm{HN}\end{array}$} & \multicolumn{2}{|l|}{ CD-sens } \\
\hline & CD63 & CD203c & CD63 & CD203c \\
\hline $\begin{array}{l}\ldots \text { with SPT } \\
(\mathrm{HN})\end{array}$ & $\begin{array}{l}0.561 \\
\quad p<0.001\end{array}$ & $\begin{array}{l}0.620 \\
\quad p<0.001\end{array}$ & $0.064 ;$ NS & $\begin{array}{l}0.389 \\
p=0.003\end{array}$ \\
\hline \multicolumn{5}{|c|}{....with IgE values } \\
\hline Total-IgE & $\begin{array}{l}0.203 \\
p=0.113\end{array}$ & $0.245 ;$ NS & $-0.262 ;$ NS & $-0.020 ;$ NS \\
\hline slgE rBet v 1 & $\begin{array}{l}0.689 \\
\quad p<0.001\end{array}$ & $\begin{array}{l}0.777 \\
p<0.001\end{array}$ & $0.203 ;$ NS & $\begin{array}{l}0.539 \\
p<0.001\end{array}$ \\
\hline $\begin{array}{l}\text { slgE rCor } \\
\text { a } 1\end{array}$ & $\begin{array}{l}0.690 \\
\quad p<0.001\end{array}$ & $\begin{array}{l}0.754 \\
\quad p<0.001\end{array}$ & $0.208 ;$ NS & $\begin{array}{l}0.541 \\
\quad p<0.001\end{array}$ \\
\hline
\end{tabular}

Correlation between CD63 and CD203c expression values at $0.01 \mu \mathrm{g} / \mathrm{ml}: 0.784$; $\mathrm{p}<0.001$ and $C D$-sens: $0.561 ; \mathrm{p}<0.001$

Spearman correlation coefficient ( $r$; v value) of BAT values at $0.01 \mu \mathrm{g} / \mathrm{ml} \mathrm{HN}$ concentration and CD-sens for CD63 and CD203c with skin prick test (SPT) for hazelnut (HN), total and slgE levels

In this study, measuring CD203c expression was superior to CD63 when sensitized and symptomatic groups were considered (Fig. 2c, d; Table 2). However, with the BAT, we were not able to distinguish a patient with systemic reactions from a patient with OAS. The ability to differentiate between the symptom severities was limited either by the diagnostic assessment, which was based on clinical history, or by the Cor-a-1-based HN allergy in our cohort. It is known that Cor a 1 can induce severe allergic symptoms but may require the intake of larger amounts of the Bet v 1 homologues such as it is the case for Gly $\mathrm{m} 4$ in soy-containing dietary food products, or it may need allergen-protective matrix effects [12]. The biological activity of Cor a 1 might be very high in birch pollen endemic regions, [25] thus, the difference between OAS and more severe allergic reactions are not detected in the BAT.

CD203c as well as CD63 expression values at a HN concentration of $0.01 \mu \mathrm{g} / \mathrm{ml}$ correlated with the SPT and sIgE results (Table 3 ). Considering CD-sens, a correlation was detected for CD203c but not CD63. Thus, the BAT can be used as a functional assay to detect sensitization status, which had been shown previously [26]. However, in our hands, CD203c seems to be superior compared to CD63. Moreover, the BAT has the potential to more closely resemble the clinical phenotype of patients [27]. In previous studies, we have shown the usefulness of CD203c expression in demonstrating differences of the allergenicity between native and roasted HN extracts [2] and between two different tomato cultivars [18]. Previous reports, where CD63 and CD203c expression were analyzed in combination, support our findings [28-30]. The data of Wanich et al. [28] indicate less basophil activity in tolerant versus symptomatic cow's milk-allergic subjects. The BAT could also differentiate between tolerant and symptomatic peanut-sensitized subjects [30]. Santos et al. [29] demonstrated the utility of basophils as biomarkers for severity and threshold of allergic reaction in a pediatric cohort suffering from peanut allergy. Whether a combined analysis is suitable is a matter of discussion due to different expression kinetics [16, 31].

Differences regarding kinetics might be a reason for the findings when comparing CD63 and CD203c in our cohort. The maximal up-regulation of CD63 occurs within 25-30 min, whereas CD203c requires only 10-20 min [32]. The stimulation time used in this study averaged $15 \mathrm{~min}$ to capture both markers.

For practical reasons, basophil activation experiments should be restricted to a single allergen concentration $[16,32]$. However, an individual, highly heterogeneous basophil response has been described previously [33] and was also present in our cohort. Thus, a single allergen concentration is not sufficient to analyze basophil responses.

The BAT offers many advantages in food allergy. In contrast to sIgE, the BAT provides a biological readout [34]. A wide range of food, raw material, purified or recombinant allergens can be analyzed [35]. Unspecific positive reactions are less frequent in BAT compared to SPT. In addition, SPT bears the risk of sensitization [36]. Moreover, basophils can be analyzed even when the subject receives anti-allergic treatment [37]. However, other 
studies demonstrated an inhibitory effect of immunosuppressants such as cyclosporin A [38] or other drugs (statins) [39]. Therefore, a careful history regarding a possible drug intake is mandatory if a BAT is considered.

The diagnosis of food allergy is mainly based on patient history, analysis of IgE and/or SPT, ideally combined with DBPCFC, which is still the gold standard in the diagnosis of food allergy [11, 12]. However, DBPCFC is expensive and time-consuming for both the physician and the patient and is often refused by the patient. Additionally, there is a risk for patients to experience severe allergic reactions. Thus, in this study, a detailed case history including allergy-focused diet history assessment was used to define the status of $\mathrm{HN}$ allergy, which had previously been shown to have a high diagnostic values $[40,41]$.

However, an ex vivo but highly diagnostic method to reliably predict clinical reactivity is desirable. Neither the presence of sIgE in the circulation nor the presence of biologically active IgE on mast cells are suitable to differentiate between sensitization and clinical allergy [35], with perhaps the exception of Cor a 9 and Cor a 14 in children cohorts [41, 42]. For both allergens, an age-dependent variability seems to exist, as allergic adults are less sensitized to both components [43]. In our cohort, no sensitization to Cor a 9, Cor a 11 or Cor a 14 was found. Thus, in our cohort Cor a 1 is the predominate allergen. The sIgE to Cor a 1 was lower in the sensitized group compared to the symptomatic groups (significant for OAS group), which was more pronounced when calculated as a sIgE/ total-IgE-ratio. Glaumann et al. [19] investigated the utility of BAT in an Ara h 8-sensitized cohort. Ara h 8 is a Bet $\mathrm{v} 1$ homologue in peanut like Cor a 1 in HN. They could show that the BAT adds safety information if sIgE and DBPCFC results were controversial. Thus, the BAT might be useful if clinical history, SPT and/or sIgE measurement are inconsistent or in addition an oral food challenge is not possible [42]. Furthermore, the use of recombinant allergens in the BAT might have added value in discriminating between clinically relevant and mere sensitization $[44,45]$ and should be considered in future studies.

Most BAT protocols are not standardized. For the future, protocol optimization is required and should consider preanalytical conditions and well-defined flow cytometry gating protocols [46]. The timeframe of analysis is relevant as well; ideally, the BAT should be performed immediately after a blood draw $[37,46]$ but at least within $24 \mathrm{~h}$, as longer storage time can lead to a loss of basophil reactivity and false negative results [37].

A weakness of this study is the small number of analyzed subjects and the results should be regarded explorative. However, we were able to differentiate between non-allergic, sensitized and symptomatic subjects. The inclusion of $\mathrm{HN}$-sensitized subjects is a strength of the study, as such a group is essential to validate a diagnostic test in allergy [33]. Still, due to the small sample size, necessary diagnostic parameters (sensitivity, specificity, positive and negative predictive value) were not calculated. Furthermore, the diagnosis of a symptomatic HN allergy was based on clinical history, as mentioned above. Moreover, we did not prove that the activation of the basophils with the $\mathrm{HN}$ extract was exclusively via crosslinking surface-bound sIgE against $\mathrm{HN}$ (Cor a 1) or via cross-reaction of surface-bound sIgE against birch homologues (Bet v 1).

The sensitivity and specificity of the BAT is influenced by the rate of non-responders. These patients do not show activation after anti-IgE stimulation [33]. The reason for this non-responsiveness of the basophils is a selective decrease in Syk expression, which is a downstream event of FceRI activation [33]. The proportion of non-responders depends on the BAT protocol and varies between 5 and 25\% [32, 33]. In this study, 3.25\% of patients when considering both expression markers and up to $6.5 \%$ of patients when considering only CD63 expression were non-responders.

\section{Conclusion}

In summary, the CD203c-based BAT differentiates between sensitization and clinically relevant allergy in $\mathrm{HN}$ sensitized individuals. The use of a given allergen extract at several concentrations is required, and the development of standardized protocols are needed to compare results obtained from different cohorts and allergens.

\section{Additional file}

Additional file 1: Figure S1. Dose-response curves for CD203c (left) and CD63 (right) expression for the four different groups (a-d). Net values without normalization to anti-IgE are shown as median with interquartile range (IQR). (+) anti-IgE (-) medium.

\section{Abbreviations}

BAT: basophil activation test; $C D$-max: the maximal percentage of surface marker up-regulation at one allergen dose; CD-sens: basophil allergen threshold sensitivity; DBPCFC: double-blind, placebo-controlled oral food challenges; EC: effective concentration; HN: hazelnut; IgE: immunoglobulin E; slgE: specific immunoglobulin E; OAS: oral allergy symptom; SPT: skin prick test.

\section{Authors' contributions}

MW and SD designed the study; BL and SD performed the statistical analyses; BL performed the basophil activation test and wrote the first draft of the manuscript; SD and MW provided laboratory assistance; SV performed slgE testing for Cor a 9, Cor a 11 and Cor a 14; SV and MW critically reviewed the analyses and edited the manuscript; SD edited and formatted the final version of the manuscript. All authors read and approved the final manuscript. 


\author{
Author details \\ ${ }^{1}$ Department of Dermatology and Allergology, Allergy-Center-Charité, Charité \\ - Universitätsmedizin Berlin, Charitéplatz 1, 10117 Berlin, Germany. ${ }^{2}$ Paul- \\ Ehrlich-Institut, Langen, Germany.
}

\section{Acknowledgements}

We thank Thermo Fisher Scientific for performing the slgE-testing. The authors also thank Dennis Ernst (technician) for supporting our lab work and for his assistance and help in conducting the flow cytometric measurements.

\section{Competing interests}

The authors declare that they have no competing interests.

\section{Availability of data and materials}

The dataset supporting the conclusions of this article is filed as "Lötzsch_Dölle sav" in the repository at the institution of the second author SD.

\section{Ethics approval and consent to participate}

Ethical approval was obtained from the ethics committee of Charité (EA-No.: 1832/Si.258). All subjects had to sign written informed consent before any study procedure was applied (a statement is included in the "Methods" section).

Received: 15 June 2016 Accepted: 18 November 2016

Published online: 13 December 2016

\section{References}

1. Burney PG, Potts J, Kummeling I, Mills EN, Clausen M, Dubakiene R, et al. The prevalence and distribution of food sensitization in European adults. Allergy. 2014;69:365-71.

2. Worm M, Hompes S, Fiedler EM, IIIner AK, Zuberbier T, Vieths S. Impact of native, heat-processed and encapsulated hazelnuts on the allergic response in hazelnut-allergic patients. Clin Exp Allergy. 2009;39:159-66.

3. Katelaris $\mathrm{CH}$. Food allergy and oral allergy or pollen-food syndrome. Curr Opin Allergy Clin Immunol. 2010;10:246-51.

4. Flinterman $A E$, Akkerdaas $\mathrm{JH}$, Knulst AC, van Ree R, Pasmans SG. Hazelnut allergy: from pollen-associated mild allergy to severe anaphylactic reactions. Curr Opin Allergy Clin Immunol. 2008;8:261-5.

5. Le TM, van Hoffen E, Lebens AF, Bruijnzeel-Koomen CA, Knulst AC. Anaphylactic versus mild reactions to hazelnut and apple in a birchendemic area: different sensitization profiles? Int Arch Allergy Immunol. 2013;160:56-62

6. Schocker F, Luttkopf D, Scheurer S, Petersen A, Cistero-Bahima A, Enrique $E$, et al. Recombinant lipid transfer protein Cor a 8 from hazelnut: a new tool for in vitro diagnosis of potentially severe hazelnut allergy. J Allergy Clin Immunol. 2004:113:141-7.

7. Lauer I, Foetisch K, Kolarich D, Ballmer-Weber BK, Conti A, Altmann F, et al. Hazelnut (Corylus avellana) vicilin Cor a 11: molecular characterization of a glycoprotein and its allergenic activity. Biochem J. 2004;383:327-34.

8. Roux KH, Teuber SS, Sathe SK. Tree nut allergens. Int Arch Allergy Immunol. 2003;131:234-44

9. Worm M, Moneret-Vautrin A, Scherer K, Lang R, Fernandez-Rivas M, Cardona V, et al. First European data from the network of severe allergic reactions (NORA). Allergy. 2014:69:1397-404.

10. Grabenhenrich LB, Dölle S, Moneret-Vautrin A, Kohli A, Lange L, Spindler T, et al. Anaphylaxis in children and adolescents: the European Anaphylaxis Registry. J Allergy Clin Immunol. 2016;137:1128-37.

11. Muraro A, Werfel T, Hoffmann-Sommergruber K, Roberts G, Beyer K, Bindslev-Jensen C, et al. EAACI food allergy and anaphylaxis guidelines: diagnosis and management of food allergy. Allergy. 2014;69:1008-25.

12. Worm M, Reese I, Ballmer-Weber B, Beyer K, Bischoff SC, Claßen M, et al. Guidelines on the management of IgE-mediated food allergies. S2K-Guidelines of the German Society for Allergology and Clinical Immunology (DGAKI) in collaboration with the German Medical Association of Allergologists (AeDA), the German Professional Association of Pediatricians (BVKJ), the German Allergy and Asthma Association (DAAB), German Dermatological Society (DDG), the German Society for Nutrition
(DGE), the German Society for Gastroenterology, Digestive and Metabolic Diseases (DGVS), the German Society for Oto-Rhino-Laryngology, Head and Neck Surgery, the German Society for Pediatric and Adolescent Medicine (DGKJ), the German Society for Pediatric Allergology and Environmental Medicine (GPA), the German Society for Pneumology (DGP), the German Society for Pediatric Gastroenterology and Nutrition (GPGE), German Contact Allergy Group (DKG), the Austrian Society for Allergology and Immunology (ÖGAI), German Professional Association of Nutritional Sciences (VDOE) and the Association of the Scientific Medical Societies Germany (AWMF). Allergo J Int. 2015;24:256-93.

13. Muraro A, Dubois AE, DunnGalvin A, Hourihane JO, de Jong NW, Meyer R, et al. EAACI food allergy and anaphylaxis guidelines. Food allergy healthrelated quality of life measures. Allergy. 2014;69:845-53.

14. Lieberman JA, Sicherer SH. Diagnosis of food allergy: epicutaneous skin tests, in vitro tests, and oral food challenge. Curr Allergy Asthma Rep. 2011:11:58-64.

15. Le TM, Lindner TM, Pasmans SG, Guikers CL, van Hoffen E, BruijnzeelKoomen CA, et al. Reported food allergy to peanut, tree nuts and fruit: comparison of clinical manifestations, prescription of medication and impact on daily life. Allergy. 2008;63:910-6.

16. Ebo DG, Sainte-Laudy J, Bridts CH, Mertens CH, Hagendorens MM, Schuerwegh AJ, et al. Flow-assisted allergy diagnosis: current applications and future perspectives. Allergy. 2006;61:1028-39.

17. Heinzerling $L$, Burbach $G$, van Cauwenberge $P$, Papageorgiou P, Carlsen $\mathrm{KH}$, Lodrup Carlsen KC, et al. Establishing a standardized quality management system for the European Health Network GA2LEN. Allergy. 2010:65:743-52.

18. Dölle S, Lehmann K, Schwarz D, Weckwert W, Scheler C, George E, et al. Allergenic activity of different tomato cultivars in tomato allergic subjects. Clin Exp Allergy. 2011:41:1643-52.

19. Glaumann S, Nilsson C, Johansson SG, Asarnoj A, Wickman M, Borres MP, et al, Evaluation of basophil allergen threshold sensitivity (CD-sens) to peanut and Ara h 8 in children lgE-sensitized to Ara h 8. Clin Mol Allergy. 2015:13:5.

20. Abuaf N, Rostane H, Rajoely B, Gaouar H, Autegarden JE, Leynadier F, et al. Comparison of two basophil activation markers CD63 and CD203C in the diagnosis of amoxicillin allergy. Clin Exp Allergy. 2008;38:921-8.

21. Potapinska O, Gorska E, Zawadzka-Krajewska A, Kulus M, Wasik M, Demkow U. The usefulness of CD203c expression measurement on basophils after activation with grass pollen and Dermatophagoides pteronyssinus antigens. Preliminary study. Pneumonol Alergol Pol. 2009;77:138-44.

22. Boumiza R, Monneret G, Forissier MF, Savoye J, Gutowski MC, Powell WS, et al. Marked improvement of the basophil activation test by detecting CD203c instead of CD63. Clin Exp Allergy. 2003;33:259-65.

23. Ocmant A, Mulier S, Hanssens L, Goldman M, Casimir G, Mascart F, et al. Basophil activation tests for the diagnosis of food allergy in children. Clin Exp Allergy. 2009;39:1234-45.

24. Ocmant A, Peignois Y, Mulier S, Hanssens L, Michils A, Schandene L. Flow cytometry for basophil activation markers: the measurement of CD203C up-regulation is as reliable as CD63 expression in the diagnosis of cat allergy. J Immunol Methods. 2007;320:40-8.

25. Blanc F, Bernard H, Ah-Leung S, Przybylski-Nicaise L, Skov PS, Purohit A, et al. Further studies on the biological activity of hazelnut allergens. Clin Transl Allergy. 2015;5:26.

26. Nilsson N, Nilsson C, Hedlin G, Johansson SG, Borres MP, Nopp A. Combining analyses of basophil allergen threshold sensitivity, CD-sens, and IgE antibodies to hydrolyzed wheat, omega- 5 gliadin and timothy grass enhances the prediction of wheat challenge outcome. Int Arch Allergy Immunol. 2013;162:50-7.

27. Santos AF, Lack G. Basophil activation test: food challenge in a test tube or specialist research tool? Clin Transl Allergy. 2016;6:10.

28. Wanich N, Nowak-Wegrzyn A, Sampson HA, Shreffler WG. Allergen-specific basophil suppression associated with clinical tolerance in patients with milk allergy. J Allergy Clin Immunol. 2009;123(789-94):e20.

29. Santos AF, Du Toit G, Douiri A, Radulovic S, Stephens A, Turcanu V, et al. Distinct parameters of the basophil activation test reflect the severity and threshold of allergic reactions to peanut. J Allergy Clin Immunol. 2015;135:179-86.

30. Santos AF, Douiri A, Becares N, Wu SY, Stephens A, Radulovic S, et al. Basophil activation test discriminates between allergy and tolerance in peanut-sensitized children. J Allergy Clin Immunol. 2014;134:645-52. 
31. MacGlashan D Jr. Expression of CD203C and CD63 in human basophils: relationship to differential regulation of piecemeal and anaphylactic degranulation processes. Clin Exp Allergy. 2010;40:1365-77.

32. De Week AL, Sanz ML, Gamboa PM, Aberer W, Bienvenu J, Blanca M, et al. Diagnostic tests based on human basophils: more potentials and perspectives than pitfalls. II. Technical issues. J Investig Allergol Clin Immunol. 2008;18:143-55.

33. Kleine-Tebbe J, Erdmann S, Knol EF, MacGlashan DW Jr, Poulsen LK, Gibbs BF. Diagnostic tests based on human basophils: potentials, pitfalls and perspectives. Int Arch Allergy Immunol. 2006;141:79-90.

34. Falcone $\mathrm{FH}, \mathrm{Knol} \mathrm{EF}$, Gibbs BF. The role of basophils in the pathogenesis of allergic disease. Clin Exp Allergy. 2011;41:939-47.

35. Asero R, Ballmer-Weber BK, Beyer K, Conti A, Dubakiene R, FernandezRivas $M$, et al. IgE-mediated food allergy diagnosis: current status and new perspectives. Mol Nutr Food Res. 2007;51:135-47.

36. Ruëff F, Bergmann KC, Brockow K, Fuchs T, Grübl A, Jung K, et al. Hauttests zur Diagnostik von allergischen Soforttypreaktionen. Allergo J. 2010;19:402-15.

37. Sturm EM, Kranzelbinder B, Heinemann A, Groselj-Strele A, Aberer W, Sturm GJ. CD203c-based basophil activation test in allergy diagnosis: characteristics and differences to CD63 upregulation. Cytom B Clin Cytom. 2010;78:308-18

38. Majlesi Y, Samorapoompichit P, Hauswirth AW, Schernthaner GH, Ghannadan $M$, Baghestanian $M$, et al. Cerivastatin and atorvastatin inhibit IL-3-dependent differentiation and IgE-mediated histamine release in human basophils and downmodulate expression of the basophil-activation antigen CD203c/E-NPP3. J Leukoc Biol. 2003;73:107-17.

39. Hauswirth AW, Natter S, Ghannadan M, Majlesi Y, Schernthaner $\mathrm{GH}$, Sperr WR, et al. Recombinant allergens promote expression of CD203C on basophils in sensitized individuals. J Allergy Clin Immunol. 2002;110:102-9.
40. Skypala IJ, Calderon MA, Leeds AR, Emery P, Till SJ, Durham SR. Development and validation of a structured questionnaire for the diagnosis of oral allergy syndrome in subjects with seasonal allergic rhinitis during the UK birch pollen season. Clin Exp Allergy. 2011;41:1001-11.

41. Masthoff $L J$, van Hoffen E, de Reus A, Boonacker CW, Bruijnzeel-Koomen CA, Pasmans SG, et al. Hazelnut allergy differs between children and adults in frequency of severity, aetiology and relevance of diagnostic parameters. Clin Exp Allergy. 2014;44:1539-45.

42. Brandström J, Nopp A, Johansson SG, Lilja G, Sundqvist AC, Borres MP, et al. Basophil allergen threshold sensitivity and component-resolved diagnostics improve hazelnut allergy diagnosis. Clin Exp Allergy. 2015;45:1412-8.

43. Masthoff $L J$, Mattsson L, Zuidmeer-Jongejan L, Lidholm J, Andersson K, Akkerdaas $\mathrm{JH}$, et al. Sensitization to Cor a 9 and Cor a 14 is highly specific for a hazelnut allergy with objective symptoms in Dutch children and adults. J Allergy Clin Immunol. 2013;132:393-9.

44. van Erp FC, Knol EF, Pontoppidan B, Meijer Y, van der Ent CK, Knulst AC. The IgE and basophil responses to Ara h 2 and Ara h 6 are good predictors of peanut allergy in children. J Allergy Clin Immunol. 2016. doi:10.1016/j.jaci.2016.06.041.

45. Faber MA, Van Gasse AL, Decuyper II, Uyttebroek A, Sabato V, Hagendorens $M M$, et al. IgE-reactivity profiles to nonspecific lipid transfer proteins in a northwestern European country. J Allergy Clin Immunol. 2016. doi:10.1016/j.jaci.2016.06.016.

46. Chirumbolo S. Basophil activation test in allergy: time for an update? Int Arch Allergy Immunol. 2012;158:99-114.

\section{Submit your next manuscript to BioMed Central and we will help you at every step:}

- We accept pre-submission inquiries

- Our selector tool helps you to find the most relevant journal

- We provide round the clock customer support

- Convenient online submission

- Thorough peer review

- Inclusion in PubMed and all major indexing services

- Maximum visibility for your research

Submit your manuscript at www.biomedcentral.com/submit 